\title{
ARCHIMEDEAN, SEMIPERFECT \\ AND $\pi$-REGULAR LATTICE-ORDERED ALGEBRAS WITH POLYNOMIAL CONSTRAINTS ARE $f$-ALGEBRAS
}

\author{
STUART A. STEINBERG
}

\begin{abstract}
It is shown that a lattice-ordered algebra is embeddable in a product of totally ordered algebras provided (i) it is archimedean, contains a left superunit which is an $f$-element, and satisfies a polynomial identity $p(x) \geqslant 0$ or $f(x, y) \geqslant 0$ (for suitable $f(x, y)$ ); or (ii) it is unital, and semiperfect, $\pi$-regular, or left $\pi$-regular, and some power of each element is positive.
\end{abstract}

A torsion-free lattice-ordered algebra $R$ over the commutative unital totally ordered domain $F$ is called an l-algebra if for all $r, s \in R^{+}=\{r \in R: r \geqslant 0\}$ and $\alpha \in F^{+}$,

$$
r \wedge s=0 \text { implies } \alpha r \wedge s=0 .
$$

Let

$$
T=\{r \in R: u \wedge v=0 \text { implies }|r| u \wedge v=u|r| \wedge v=0\} .
$$

Then $T$ consists of the f-elements of $R ; T$ is a convex $l$-subalgebra of $R$ which contains 1 , if $1 \in R^{+}$; and $R$ is an $f$-algebra precisely when $T=R$.

The variety of $f$-rings, which was introduced by Birkhoff and Pierce in [1], has been the most extensively studied class of $l$-rings. This is because an $f$-algebra is a subdirect product of a family of totally ordered algebras, and, hence, computations in $f$-algebras can frequently be reduced to the totally ordered case. However, larger classes and varieties of $l$-algebras have been studied by Birkhoff and Pierce [1], Diem [2], Shyr and Viswanathan [3], and Steinberg [4-7].

The $l$-algebra $R$ is $l$-prime if the product of two of its nonzero $l$-ideals is nonzero, and an $l$-domain if the product of two nonzero positive elements is nonzero. $R$ is reduced if $a^{2}=0$ implies $a=0$. In [2, p. 79] Diem asked if an $l$-prime $l$-ring $R$ in which the square of every element is positive must be an $l$-domain. In [7] we have shown that $R$ must be a domain if it is unital or the left and right annihilator ideals of $T$ vanish. More generally, the same conclusion follows if the identity $x^{2} \geqslant 0$ (actually, $\left(x^{2}\right)^{-}=0$ ) is replaced by more general polynomial constraints. Let $F[x, y]$ be the free noncommutative $F$-algebra in two variables $x$ and $y$. A polynomial $f(x, y) \in F[x, y]$ is nice if

$$
f(x, y)=-g(x, y)+p(y)+h(x, y)
$$

Received by the editors July 12, 1982.

1980 Mathematics Subject Classification. Primary 06F25; Secondary 16A86.

Key words and phrases. Lattice-ordered ring, totally ordered ring, polynomial constraint. 
where $0 \neq g(x, y)$ is of degree 1 in $x$ and has all its coefficients positive, and $h(x, y)=0$ or each of its monomials has degree at least 2 in $x . f(x, y)$ is left (right) nice if $g(x, y)$ has a monomial which begins (ends) with $x$, and is $k$-nice if $h(x, y) \in F\left[x^{k}, y\right]$. For example, $-x$ and $(x-y)^{2}$ are left and right 2-nice polynomials. From [7] we have the

THEOREM. Let $R$ be an l-prime l-algebra over the totally ordered domain $F$.

(1) If $1 \in R^{+}$and if $u \wedge v=0$ implies there is a nice polynomial $f(x, y) \in F[x, y]$ with $f(u, v) \geqslant 0$, then $R$ is an l-domain.

(2) Each of the following conditions implies that $R$ is a reduced l-domain.

(a) $1 \in R$ and for each invertible element $u \in R$ there is a polynomial $p(x) \in F[x]$ with $p(u) \geqslant 0$ and $0 \neq p^{\prime}(1) \in R^{+}\left(p^{\prime}(x)\right.$ is the derivative of $\left.p(x)\right)$.

(b) If $r \in R$ and $r T=0$ or $T r=0$, then $r=0$; and there is a right and left $k$-nice polynomial $f(x, y) \in F[x, y]$ (with $k \geqslant 2$ ) such that $R$ satisfies $f\left(x, y^{+}\right)^{-}=0$.

(3) If $F$ is a field and $1 \in R^{+}$, then each of the following implies that $R$ is a domain.

(a) For each $r \in R$ there is a polynomial $p(x) \in x F[x]$ with $p(r) \in R^{+}, p(1) p^{\prime}(1)$ $\neq 0$, and $p^{\prime}(1) \in R^{+}$.

(b) $R$ satisfies the identity $p(x)^{+} p(x)^{-}=0$ where $p(x) \in F[x]$ has only odd terms and $p(1) p^{\prime}(1) \neq 0$.

In this note we investigate $l$-algebras with such polynomial constraints. In particular, we show that the squares positive hypothesis in [4 and 6] can be relaxed; that is, $l$-algebras with certain constraints that are archimedean, semiperfect, algebraic, $\pi$-regular or left $\pi$-regular must be $f$-algebras.

If $r$ and $s$ are two elements of the $l$-algebra $R$, then $r$ is infinitely smaller than $s$ with respect to $F$, written $r \ll s$, if $\alpha|r| \leqslant|s|$ for each $\alpha \in F$. $R$ is archimedean over $F$ if $r \ll s$ implies $r=0$. $R$ is a PPI l-algebra over $F$ if $R$ satisfies the identity $f(x, y)^{-}=0$ where $f(x, y) \in F[x, y]$ and $f(x, y) \notin F$. By a left superunit $e$ in $R$ we mean an element $e \in R^{+}$such that $e x \geqslant x$ for each $x$ in $R^{+}$. The element $a \in R$ is a left f-element if $b \wedge c=0$ implies $|a| b \wedge c=0$, and a weak order unit if $|a| \wedge b=0$ implies $b=0$. For notational convenience we note that $F[x, y]$ is an $l$-algebra with positive cone $F^{+}[x, y]$, and we will denote the positive part, negative part and absolute value of $f(x, y)$ by $f^{+}(x, y), f^{-}(x, y)$ and $|f|(x, y)$, respectively.

1. Archimedean $l$-algebras. To show that archimedean PPI $l$-algebras are $f$-algebras we require two lemmas.

LEMMA 1. Let $a, e \in R^{+}$and suppose that there exists $p(x)=\alpha_{0}+\alpha_{1} x$ $+\cdots+\alpha_{n} x^{n} \in F[x]$ of degree $n \geqslant 1$ with $\alpha_{n}>0$ and $0 \leqslant p(\alpha e-a)$ for each $\alpha$ in $a$ cofinal subset of $F^{+}$. Suppose also that

(a) There exist $0<\delta_{1}, \delta_{2} \in F$ with

$$
\delta_{1} a \leqslant \delta_{2} \sum_{i+j=n-1} e^{i} a e^{j},
$$

(b) $a \wedge e^{n}=0$,

(c) $a \wedge e^{n-1}=0$ if $\alpha_{n-1}>0$.

Then there exists $0<\rho \in F$ and $q(x, y) \in F^{+}[x, y]$ with $\rho a \ll q(a, e)$. 
Proof. We assume that $n \geqslant 2$. The coefficient of $\alpha^{k}$ in $p(\alpha e-a)$ comes from $\alpha_{k}(\alpha e-a)^{k}+\alpha_{k+1}(\alpha e-a)^{k+1}+\cdots+\alpha_{n}(\alpha e-a)^{n}$ and is

$$
\alpha_{k} e^{k}+\sum_{m \geqslant k+1}(-1)^{m-k} \alpha_{m} \sum_{\substack{i_{1}+\cdots+i_{t}=k \\ j_{1}+\cdots+j_{t}=m-k}} e^{i_{1}} a^{j_{1}} \cdots e^{i_{t}} a^{j_{t}}=\alpha_{k} e^{k}+\mathfrak{e}_{k}(a, e) .
$$

So

$$
\begin{aligned}
0 & \leqslant p(\alpha e-a)=p(-a)+\sum_{k=1}^{n}\left[\alpha_{k} e^{k}+\Theta_{k}(a, e)\right] \alpha^{k} \\
& =p(-a)+[p(\alpha e)-p(0)]+\sum_{k=1}^{n-2} \vartheta_{k}(a, e) \alpha^{k}-\alpha_{n} \alpha^{n-1} \sum_{i+j=n-1} e^{i} a e^{j}
\end{aligned}
$$

Thus,

$$
\begin{aligned}
0 & \leqslant \alpha_{n} \delta_{1} \alpha^{n-1} a \\
& \leqslant \delta_{2}\left[h^{+}(a)+\sum_{k=1}^{n-2}\left(\alpha_{k}^{+} e^{k}+\vartheta_{k}^{+}(a, e)\right) \alpha^{k}+\alpha_{n-1}^{+} \alpha^{n-1} e^{n-1}+\alpha_{n}^{+} \alpha^{n} e^{n}\right],
\end{aligned}
$$

where $h(x)=p(-x)$. So if $\alpha \geqslant 1$,

$$
0 \leqslant \alpha\left(\delta_{1} \alpha_{n} a\right) \leqslant \delta_{2}\left[h^{+}(a)+\sum_{k=1}^{n-2}\left(\alpha_{k}^{+} e^{k}+\vartheta_{k}^{+}(a, e)\right)+\alpha_{n-1}^{+} \alpha e^{n-1}+\alpha_{n} \alpha^{2} e^{n}\right]
$$

and by (b) and (c),

$$
0 \leqslant \alpha \rho a \leqslant q(a, e) \quad \text { with } \rho=\alpha_{n} \delta_{1} .
$$

COROLlARY. The following statements are equivalent for the archimedean l-algebra $R$ over $F$.

(a) $R$ is an f-algebra.

(b) For each $a \in\left\{u^{+} v^{+} \wedge v^{-}, v^{+} u^{+} \wedge v^{-}: u, v \in R\right\}$ there exists $p(x) \in F[x]$ and a left f-element $e \geqslant 0$ such that for all $\alpha \in F^{+}$

$$
(a \wedge e) \vee(e a-a)^{-} \vee p(\alpha e-a)^{-}=0 .
$$

LEMmA 2. Let $f(x, y) \in F[x, y]$ be a polynomial such that $f^{-}(x, y)$ has a monomial of positive degree in $x$ whose degree in $y$ exceeds the degree of $f^{+}(x, y)$ in $y$. Suppose that $a, e \in R^{+}$with $a \leqslant e a$ and $f(a, \alpha e) \geqslant 0$ for each $\alpha$ in a cofinal subset of $F^{+}$. Then there exist $0<\rho \in F$ and $q(x, y) \in F^{+}[x, y]$ with $\rho a^{n} e^{t} \ll q(a, e)$ for some integers $n \geqslant 1$ and $t \geqslant 0$.

Proof. Write $f(x, y)=-\rho m(x, y)+h(x, y)$, where $\rho>0$ and $m(x, y)$ is a monomial whose degree in $y$ exceeds the degree in $y$ of $h^{+}(x, y)$. Since $a^{i} \leqslant e^{k} a^{i}$ if $i \geqslant 1$ and $k \geqslant 0, a^{n} e^{t} \leqslant m(a, e)$ where $n \geqslant 1$ is the degree of $x$ in $m(x, y)$ and $m(x, y)$ ends in $y^{t}$. If $m(x, y)$ has degree $s$ in $y$, then $f(a, \alpha e) \geqslant 0$ implies

$$
0 \leqslant \rho \alpha^{s} a^{n} e^{t} \leqslant \rho m(a, \alpha e) \leqslant h(a, \alpha e) \leqslant h^{+}(a, \alpha e) .
$$

If $\alpha \geqslant 1$, then

$$
\rho \alpha a^{n} e^{t} \leqslant \alpha^{1-s} h^{+}(a, \alpha e) \leqslant h^{+}(a, e)=q(a, e),
$$

since $s>$ degree of $y$ in $h^{+}(x, y)$. 
The equivalence of (a) and (b) in the following theorem is given in [6, Corollary 4 , p. 206] for the case $p(x)=x^{2}$. Also, it is shown in Theorem 8 of [6] that $e$ is a weak order unit precisely when $R$ satisfies $x^{+} x^{-}=0$. Thus, the equivalence of (a) and (b) follows from Lemma 1.

THEOREM 1. Let $R$ be an archimedean l-algebra over $F$ and suppose that $R$ has a left superunit e which is an f-element. The following statements are equivalent.

(a) $R$ is an f-algebra.

(b) $R$ is a PPI l-algebra and satisfies the identity $p(x)^{-}=0$ for some $p(x) \in F[x]$.

(c) $R$ is a PPI l-algebra and satisfies the identity $f(x, y)^{-}=0$, where $f(x, y)=$ $-g(x, y)+p(y)+h(x, y)$ is a right $k$-nice polynomial with $k \geqslant 2$, and $y$ has higher degree in $g(x, y)$ than in $h^{+}(x, y)$.

(d) $R$ satisfies $f\left(x^{+}, x^{-}\right)^{-}=0$ where $f(x, y)$ is a polynomial satisfying the conditions in $(\mathrm{c})$.

Proof. (d) $\rightarrow$ (a). Let $a \wedge e=0$. Then if $\alpha \geqslant 0$,

$$
0 \leqslant g(a, \alpha e) \leqslant p(\alpha e)+h(a, \alpha e) \leqslant|p|(\alpha e)+h^{+}(a, \alpha e) .
$$

Since $g(a, \alpha e) \wedge|p|(\alpha e)=0, g(a, \alpha e) \leqslant h^{+}(a, \alpha e)$. By Lemma $2 a e^{t}=0$ and hence $a^{2}=0$ since $a \leqslant e^{t} a$. By [7, Lemma 10], $a \in T$. But $T$ is an archimedean $f$-algebra with a superunit and hence is reduced. So $e$ is a weak order unit of $R$ and by the remarks preceding the theorem, $R$ is an $f$-algebra.

Since the equivalence of (a) and (b) has already been noted and since the implications $(\mathrm{a}) \rightarrow(\mathrm{c})$ and $(\mathrm{c}) \rightarrow(\mathrm{d})$ are trivial, the proof is complete.

In view of the theorem in the introduction one might conjecture that the identity $p(x)^{-}=0$ could be localized in Theorem 1, namely, replaced by "for each $u \in R$ there exists $p(x)$ with $p(u) \geqslant 0$ ". The following example shows that this is not possible. Let $R=\mathbf{Q}(\sqrt{2})=\mathbf{Q} \oplus \mathbf{Q} \sqrt{2}$ as l-groups. Then for $b \geqslant 0$ or $b \leqslant 0, p(b) \geqslant 0$ if $p(x)=x^{2}$; and if $b=p+q \sqrt{2}$ with $p q<0$,

$$
\text { then } p(b) \geqslant 0 \text { if } p(x)=\left[\left(p^{2}+2 q^{2}\right)-x^{2}\right]^{2} \text {. }
$$

Using a polynomial $f(x, y)$ which satisfies the conditions in (c) it is possible to add the following statement as a third equivalence in the corollary.

For each $a \in\left\{u^{+} v^{+} \wedge v^{-}, v^{+} u^{+} \wedge v^{-}: u, v \in R\right\}$ there is an $f$-element $e \geqslant 0$ with

$$
(a \wedge e) \vee(e a-a)^{-} \vee f\left(a, s^{+}\right)^{-}=0
$$

for each $s$ in the convex $l$-subalgebra generated by $e$.

We also note that Diem's example [2,p. 72] shows that an archimedean $l$-domain with squares positive need not be an $f$-ring.

2. Chain conditions on the algebra. Recall that the unital $l$-ring $R$ with Jacobson radical $J$ is local if $R / J$ is a division ring, and semiperfect if $R / J$ is left artinian and idempotents may be lifted from $R / J$ to $R$. Theorem 2 below is given in [4] for the case in which $R$ has squares positive. 
Lemma 3. Let $R$ be a local l-algebra with radical J. Then $R$ is an f-algebra if and only if the inverse of each positive element is positive.

Proof. Assume that $\left(R^{+} \backslash J\right)^{-1} \subseteq R^{+}$. Let $a \in R^{+}$and put $b=a \vee 2$; and suppose that $x \wedge y=0$. If $b \notin J$, then $b^{-1} \in R^{+}$and

$$
0 \leqslant b^{-1}(b x \wedge b y) \leqslant b^{-1} b x \wedge b^{-1} b y=0 .
$$

So $b x \wedge b y=0$. If $b \in J$, then $(b-1)^{-1} \in R^{+}$and again $(b-1) x \wedge(b-1) y=0$. Thus

$$
0 \leqslant(b-1) x \wedge y \leqslant(b-1) x \wedge(b-1) y=0
$$

and so $b x \wedge y=[(b-1) x+x] \wedge y=0$. In either case

$$
0 \leqslant a x \wedge y \leqslant b x \wedge b y=0 .
$$

Similarly, $x a \wedge y=0$ and $R$ is an $f$-algebra.

THEOREM 2. Let $R$ be a unital l-ring such that for each $a \in R$ there is an integer $n \geqslant 1$ with $a^{n} \geqslant 0$. If $R$ is semiperfect, $\pi$-regular, left $\pi$-regular or an algebraic algebra over a field, then $R$ is an f-ring.

Proof. Since the idempotents of $R$ are all positive, they are central and contained in $T$. If $R$ is semiperfect, then $R / J$ is a direct sum of division rings and, hence, if $1=e_{1}+\cdots+e_{m}$ is a lifting of the orthogonal idempotents of $R / J$, then $R=R e_{1}$ $\oplus \cdots \oplus R e_{m}$ as $l$-rings. So we may assume that $R$ is local. But if $u \in R^{+}$is invertible and $u^{-n} \geqslant 0$, then $u^{-1}=u^{n-1} u^{-n} \geqslant 0$. So $R$ is an $f$-ring by Lemma 3 .

Suppose that $R$ is $\pi$-regular. So for each $a \in R$ there is an integer $t$ and $b \in R$ with $a^{t}=a^{t} b a^{t}$; hence $e=b a^{t}$ is idempotent and $R a^{t}=R e$. We may assume that $R$ is a subdirectly irreducible $l$-ring. But then $R$ is an indecomposable $l$-ring and hence $e=0$ or 1 . Since $e=1$ if and only if $a$ is a unit, the nonunits form a nil ideal. In particular, $R$ is local and hence an $f$-ring by Lemma 3 .

If $R$ is left $\pi$-regular, that is, each chain $R a \supseteq R a^{2} \supseteq \cdots$ is finite, and $a \in R^{+}$, put $b=a \vee 1$. Then for some integer $m$ and $x \in R, b^{m}=x b^{m+1}$. Thus $(1-x b) b^{m}$ $=0$. If $(1-x b)^{n} \geqslant 0$, then $(1-x b)^{n} b^{m}=0$ and hence $(1-x b)^{n}=0$ since $b \geqslant 1$. But then $x b=1-(1-x b)$ is a unit, and therefore so is $b$. Since $b, b^{-1} \in R^{+}$, as in the proof of Lemma 3, we see that $a \in T$ and hence $R$ is an $f$-ring.

Since an algebraic algebra is $\pi$-regular the proof is complete.

Let $F$ be a totally ordered field and let $F_{n}$ be the canonically ordered $n \times n$ triangular matrix $l$-algebra over $F$. So

$$
F_{n}=\left\{\left(a_{i j}\right): a_{i j}=0 \text { if } i>j\right\}
$$

and

$$
F_{n}^{+}=\left\{\left(a_{i j}\right) \in F_{n}: a_{i j} \geqslant 0 \text { for each } i \text { and } j\right\} .
$$

It can be shown that the $F$-l-algebra $R$ is isomorphic to $F_{2}$ if and only if $R$ satisfies the following three conditions:

(i) $R$ is noncommutative and 3-dimensional over $F$.

(ii) $\left\{a \in R: a^{m}=0\right\}$ is a 1-dimensional $l$-ideal.

(iii) $R$ satisfies the identity $\left(\left(x^{2}\right)^{-}\right)^{2}=0$.

Using the identity $\left(\left(x^{2}\right)^{-}\right)^{n}=0$, what is the analogous characterization of $F_{n}$ ? 


\section{REFERENCES}

1. G. Birkhoff and R. S. Pierce, Lattice-ordered rings, Ann. Acad. Brasil. Ciênc. 28 (1956), 41-69.

2. J. E. Diem, A radical for lattice-ordered rings, Pacific J. Math. 25 (1968), 71-82.

3. H. J. Shyr and T. M. Viswanathan, On the radicals of lattice-ordered rings, Pacific J. Math. 54 (1974), 257-260.

4. S. A. Steinberg, On lattice-ordered rings in which the square of every element is positive, J. Austral. Math. Soc. Ser. A 22 (1976), 362-370.

5. __ Radical theory in lattice-ordered rings, Sympos. Math. 21 (1971), 379-400.

6. __ Identities and nilpotent elements in lattice-ordered rings, Ring Theory (S. K. Jain, Ed.), Dekker, New York, 1977.

7. Unital l-prime lattice-ordered rings with polynomial constraints are domains, Trans. Amer. Math. Soc. 276 (1983), 145-164.

Department of Mathematics, University of Toledo, Toledo, Ohio 43606 